\title{
Healthcare providers: will they come to work during an influenza pandemic?
}

\author{
C. Rossow ${ }^{1}$, L. V. Ivanitskaya ${ }^{2}$, L. Fulton ${ }^{3} \&$ W. Fales ${ }^{4}$ \\ ${ }^{1}$ Governors State University, USA \\ ${ }^{2}$ Central Michigan University, USA \\ ${ }^{3}$ Texas State University, USA \\ ${ }^{4}$ Western Michigan University, USA
}

\begin{abstract}
The objective of the study was to examine available evidence on healthcare providers' willingness to report to work during a pandemic influenza and on related measures. Limited to scholarly journals, a search in nine databases produced 206 studies, 28 of which met inclusion criteria and were discussed in depth this review. Six studies posed an avian influenza scenario, four studies were conducted during the 2009/2010 global H1N1 pandemic influenza and the remaining 18 studies examined willingness to report to work under a scenario that was related to a pandemic influenza but the type of flu was under-specified. Willingness to report to work varied dramatically among the 28 studies, from a low of $23.1 \%$ to a high of $93.1 \%$. Heterogeneous methods employed by researchers make it hard to generalize and draw practical conclusions. Preliminary evidence suggests that physicians may be more willing to report to work than nurses. Given the current state of the literature and the need to estimate workforce availability for preparedness planning, worst case scenario planners may cautiously assume that 1 in 4 healthcare providers will be willing to report to work during a pandemic influenza, not accounting for those who are ill. Because physicians demonstrated higher willingness, the 1 in 4 estimate could be adjusted upward for this provider group.
\end{abstract}

Keywords: healthcare providers, willingness to report to work, influenza pandemic. 


\section{Introduction}

The 2009 H1N1 virus produced the first flu pandemic in over 40 years (CDC [1]). Between April 2009 and April 2010 the H1N1virus was responsible for an estimated 43 million to 89 million illnesses, 195,000 and 403,000 hospitalizations, and an estimated 8,870 to 18,300 deaths (CDC [1]). Though underestimated the World Health Organization (2010), published studies suggest that 20-40 percent of populations in various regions of the globe were infected (Chan [2]).

Pandemics have a long-standing history and have been recorded since the 16th century at intervals ranging between 10 and 50 years with varying severity and impact (WHO [3]). Most notable was the severe pandemic of 1918, the Spanish Flu that killed more people than the Great War, known today as World War 1 (Stanford University [4]). Caused by subtype H1N1 the 1918 pandemic was accountable for illness in approximately 20 to 40 percent of the world's population and more than 50 million deaths (USDHHS [5]). The flu was most deadly for those individuals between 20 and 40 years of age (Stanford University [4]). An estimated 28 percent of all Americans contracted the virus resulting in an estimated 675,000 deaths (Stanford University [4]). In many cities, more than half of all families had at least one victim ill with influenza (Barry [6]). Lesser pandemics followed; in1957, the Asian Flu killed approximately 70,000 Americans and the Hong Kong Flu in 1968 pursued with 34,000 deaths (USDHHS [7]).

Differences amongst outbreaks are primarily related to the severity of infection and virulence of the viruses responsible for the outbreak. The 20th and 21st century pandemics share similar characteristics. In each occurrence, approximately 30 percent of the United States population developed the affliction, with half seeking medical care (USDHS [8]). Children under age 18 typically have the highest rate of illness, morbidity and mortality. Geographically each pandemic was rapid and essentially all communities experienced out breaks (USDHS [8]).

If the outbreak is severe, as with the Spanish Flu the impact on society may include a 40 to 50 percent absentee rate among healthcare workers; an overwhelmed healthcare system; interruptions in the functions of societal infrastructure elements such as transportation, supply chains (including medical supplies), water, electricity, financial systems; and the weakening of the overall economy (AHRQ [9]). The national response and contingency plans suggest healthcare workforce shortages may well occur due to illness and mortality among the healthcare providers (OSHA [10]). In addition to illness, unwillingness to report to work during a pandemic outbreak is another factor contributing to healthcare worker shortages.

Emergency preparedness planning is dedicated to finding ways to decrease suffering and minimize the loss of life. Healthcare professionals play a critical role in the disaster response workforce. An effective plan begins with an adequate quantity of healthcare professionals who are able and willing to report to work in a pandemic influenza. Adding to the complexity is the aging 
healthcare workforce which will impact the number of available personnel physically capable of working in a disaster like environment (NCDMPH [11]). A predicted shortage of 100,000 physicians and 300,000 to 1 million nurses are projected over the next ten years (NCDMPH [11]). In addition, the number of nurses $(77 \%)$ are 50 year of age or older which will add to the severity of the shortage (NCDMPH [11]).

Present day pandemic threat is linked to an outbreak of avian influenza by the H5N1 strain of the influenza A virus (Homeland Security Council [12]). Transmitted by infected poultry, hundreds of millions of chickens, ducks, turkeys and geese have died or been culled to prevent the spread of the virus (Kilpatrick et al. [13]). A total of 608 confirmed human cases, 359 deaths were reported to the World Health Organization (WHO) between January 2003 and August 10, 2012 from a number of countries in Asia, the Near East, Africa, and Europe (WHO [14]). Currently limited to poultry workers the virus has shown an inability to spread from human to human. However, the possibility of mutations over time raises fears and apprehensions that the virus will become communicable between humans with disastrous consequences (USDHHS [5]).

An influenza pandemic will place tremendous burden on the healthcare system. In fact, as in 1918, an influenza pandemic could quickly rise to a level of a catastrophic incident resulting in mass fatalities; placing overwhelming religious, cultural, and emotional problems on local jurisdictions and the family of the victims (USDHHS [7]). Based on these characterises, federal planning estimates suggest the number of people seeking medical care may rise to 50 percent (USDHHS [7]). The demand of intensive care beds and ventilator services may increase by $25 \%$ (USDHHS [7]).

For this type of disaster response characterized by a surge of patients with symptoms, three occupational groups of healthcare providers are essential: physicians, nurses, and emergency medical personnel. Emergency and critical care physicians, critical care nurses, and paramedics are of particular importance (NCDMPH [11]). In addition, a multi-disciplinary team of doctors, nurses, pharmacists, respiratory therapists, and other healthcare professionals to maintain a continuum of care. Infrastructure support members, ancillary staff members who provide nutritional and environmental services, maintenance, and medical supplies are also essential to ensure operations (OSHA [10]).

Therefore, an effective response begins with an adequate healthcare workforce ready, able and willing to respond to the surge of patients. Reallocating healthcare professionals from non-acute care facilities, recruiting retired healthcare professionals, reserve or military medical and nursing personnel, and qualified volunteers will be essential to meet the workforce demands (AHRQ [9]).

It is imperative for workforce planning to have accurate data demonstrating healthcare providers' willingness to report to work. But what is known of healthcare professionals' willingness to report to work? Chaffee [15] presents a systematic review of 27 articles published between 1991 and 2007, identifying certain factors influencing the willingness to work. A number of disaster scenarios - weather-related, radiological, nuclear, biological, and chemical - 
were examined. Chaffee cites a biological outbreak as a significant barrier to willingness to work referring to one study that reported the lowest willingness to report to work rate that was observed in response to a scenario of a hypothetical pandemic influenza outbreak in New York City. A mere 11 percent of healthcare aides and 37 percent of registered nurses were willing to take care of patients infected with the influenza (Gershon et al. [16]). Most participants in the Gershon et al. [16] study were female, home healthcare aides, and likely lowwage earners who were concerned about child care if schools were closed and feared for personal and family safety. Chaffee recommends that future researchers enhance their measurement tools in order to build confidence in the data and utilize information for preparedness planning.

While Chaffee's review includes various natural disasters, pandemic influenza was not individually addressed. Even though some studies have attempted to answer the question of how many healthcare providers will report to work during a pandemic influenza event, no systematic review exists. This study seeks to fill this gap by reviewing studies that specifically examined healthcare providers' willingness to report to work during influenza pandemics. Furthermore, what is known of the willingness of the three provider groups emergency and critical physicians, critical nurses and paramedics - who are best able to provide care in a disaster with surge capacity? Can preparedness planning be enhanced by generalizing the results from the available studies?

\section{Method}

Nine databases were reviewed for sources: Cinahl (1950 - 2011); CSA Illumina (earliest-2011); Healthcare Reference Center (all); Health Sciences (18792011); Health Source (all); Nursing and Allied Health (all); Medline (19922011); ProQuest (all); PubMed Central (all); and Google Scholar (1950-2011) in December 2009, followed by additional searches in December 2010 and in May 2011. Keywords for search included willingness to report to work, to respond, to risk one's life and care for patients, to provide clinical services; likelihood of reporting, of working or continuing to work in a pandemic influenza; as well as barriers and strategies to enhance willingness. Article reference lists were manually examined to identify further applicable studies.

All titles and abstracts were reviewed to remove duplicates. A full review of the article was completed if it met inclusion criteria requirements: a study published in a peer-reviewed scholarly journal, study participants are healthcare providers and results are reported on willingness/likelihood to report to work during an influenza pandemic. Articles and abstracts that met the inclusion criteria $(n=206)$ were thoroughly examined in full text. Scholarly articles that presented original research were further examined utilizing methodology modified from Shi [17]. Then comparative tables were constructed demonstrating response rates, sampling methods and respondent characteristics; operational definitions of dependent variables and author-stated percentage of willingness to report to work; as well as any other significant findings and notes. The quality of the research design was analysed for each of the included studies. 


\section{Results}

\subsection{Literature search}

A systematic literature review of nine electronic data bases completed in May 2010 revealed 263 records with any of the following keywords Healthcare Providers' (HCPs') willingness to report to work, willingness to respond, likelihood of reporting to work, likelihood of working and likelihood of continuing to work during a pandemic. The process used for identifying 28 studies included in this review is summarized in figure 1. Three relevant qualitative studies were also obtained but their number was too small to conduct a separate review.

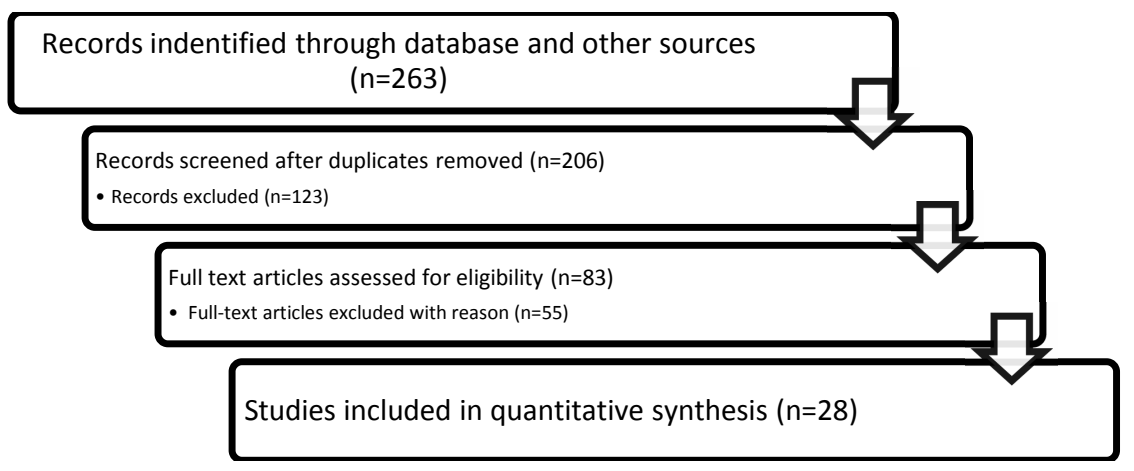

Figure 1: $\quad$ Systematic literature review process.

\subsection{Geographic location}

Most studies (15 or 53\% representing 16,847 study participants) were conducted in the United States and the remaining studies came from Australia, China, Egypt, Japan, Taiwan and the United Kingdom (14,816 study participants). Due to the limited number of studies from the same country, the only possible comparison was US vs. non-US. As shown in table 1, US average willingness to work was $68.65 \%$, as compared to $63.22 \%$ in non-US studies.

\subsection{Influenza pandemic events}

Four studies were completed during the actual H1N1 pandemic global outbreak of 2009/2010 as shown in table 1. In these studies, willingness to report to work varied from $23.10 \%$ in Wong et al. [21] to $90.10 \%$ in Martin [18], $\mathrm{M}=63.50$. Martin [18] examined factors affecting nurses' ability and willingness to work during an on-going pandemic influenza. This large study included 22,000 nurses who resided in the state of Maine, United States. Similarly, Saleh and Elshaer 
[19] conducted their study when the country of Egypt was on the edge of the pandemic. The purpose of the study was to assess the effect of the $2009 \mathrm{H} 1 \mathrm{~N} 1$ pandemic on nurses' working behaviour, identify willingness to work, concerns and persuading factors towards working during an actual pandemic. The remaining two studies were conducted in China during the second wave of the same pandemic. Ma et al. [20] assessed the knowledge and attitudes of critical care clinicians in ICUs while Wong et al. [21] explored the willingness of Hong Kong community nurses to continue to work during the H1N1 pandemic. As can be seen even from this brief description, the researchers focused on different populations (nurses vs. ICU critical care clinicians) and assessed willingness to work at different points of the global outbreak, which may account, at least in part, for an extremely wide range of findings. Nevertheless, the context in which these studies were performed - an unfolding pandemic - elevates the significance of these findings above and beyond the findings from studies that elicited responses to hypothetical scenarios.

Table 1: Author, country of origin, event, sector, respondents, and percent willingness to report.

\begin{tabular}{|c|c|c|c|c|c|c|}
\hline Study Author/Year & Country & Event & HC Sector & $\begin{array}{l}\text { HCP } \\
\text { Type }\end{array}$ & $\begin{array}{r}\text { No of } \\
\text { Respondents }\end{array}$ & $\begin{array}{r}\text { Percent } \\
\text { Willingness }\end{array}$ \\
\hline Balicer et al 2006 [32] & US & IP & Public Health & Various & 308 & 53.80 \\
\hline Balicer et al 2010 [33] & US & IP & Hospital/HCS & Various & 3,426 & 82.50 \\
\hline Barnett et al 2010 [34] & US & IP & EMS & EMS & 586 & 93.10 \\
\hline Barnett et al 2009 [28] & US & IP & Public Health & Various & 1,835 & 92.00 \\
\hline Basta et al 2009 [31] & US & IP & Public Health & Various & 2,414 & 56.20 \\
\hline Cone et al 2006 [29] & US & IP & Hospital/HCS & Various & 1,711 & 72.00 \\
\hline Cowden et al 2010 [35] & US & IP & Hospital/HCS & Various & 778 & 60.00 \\
\hline Damery et al 2009 [36] & UK & IP & Hospital/HCS & Various & 1,032 & 59.30 \\
\hline Daugherty et al 2009 [37] & US & IP & Hospital/HCS & Various & 256 & 79.00 \\
\hline Garnett et al 2009 [38] & US & IP & Hospital/HCS & Various & 2,864 & 75.60 \\
\hline Gershon et al 2010 [16] & US & IP & Home Care & Various & 384 & 27.00 \\
\hline Hope et al 2009 [39] & Australia & IP & Hospital/HCS & Nurses & 47 & 47.00 \\
\hline Hope et al 2010 [30] & Australia & IP & Hospital/HCS & Various & 868 & 67.00 \\
\hline Imai et al 2009 [40] & Japan & IP & Hospital/HCS & Various & 7,378 & 74.50 \\
\hline Irvin et al 2008 [22] & US & AI & Hospital/HCS & Various & 169 & 50.00 \\
\hline Ma et al 2011 [20] & China & H1N1 & Hospital/HCS & Various & 695 & 82.30 \\
\hline Martin 2011 [18] & US & H1N1 & Other & Nurses & 735 & 90.10 \\
\hline Martinese et al 2009 [23] & Australia & $\mathrm{AI}$ & Hospital/HCS & Various & 560 & 64.00 \\
\hline Saleh \& Elshaer 2010 [19] & Egypt & H1N1 & Hospital/HCS & Various & 266 & 58.50 \\
\hline Schechter $2007[26]$ & US & $\mathrm{AI}$ & Other & Various & 198 & 79.00 \\
\hline Seale et al 2009 [41] & Australia & IP & Hospital/HCS & Various & 894 & 83.30 \\
\hline Shabanowitz et al 2009 [24] & US & AI & Hospital/HCS & Various & 1,003 & 79.00 \\
\hline Stuart \& Gillespie 2008 [42] & Australia & IP & Hospital/HCS & Various & 1,440 & 67.00 \\
\hline Syret et al 2006 [43] & US & IP & Hospital/HCS & Various & 180 & 40.00 \\
\hline Tippett et al 2010 [44] & Australia & IP & EMS & EMS & 725 & 56.30 \\
\hline Tzeng et al 2006 [25] & Taiwan & AI & Hospital/HCS & Nurses & 225 & 57.00 \\
\hline Wong E et al 2010 [21] & China & H1N1 & Other & Nurses & 401 & 23.10 \\
\hline Wong T et al 2008 [27] & China & AI & Other & Physician & 285 & 82.50 \\
\hline
\end{tabular}


Six of the studies presented a well-defined yet a hypothetical avian influenza scenario. Irvin et al. [22] examined the willingness of hospital personnel in a Midwestern US medical center to report to work in an avian influenza and factors influencing their decisions. Martinese et al. [23] in Australia examined how an avian or pandemic influenza threat would affect hospital staff members' absenteeism while Shabanowitz et al. [24] researched willingness of providers at a US medical center. Tzeng and Yin [25] in Taiwan illustrated factors that contributed to nurses' fear about an avian influenza and willingness to care for infected patients. Schechter [26] determined the ability and willingness of the Medical Reserve Corp volunteers to work in a public health emergency (both influenza pandemic and avian flu). Wong et al. [27] examined primary care physicians in outpatient clinics for an avian influenza pandemic. In these studies mean willingness to report to work varied from $50.00 \%$ (Irvin et al. [22]) to $82.50 \%$ (Wong et al. [27]), $\mathrm{M}=68.58$.

In the remaining 18 studies, researchers either examined multiple events (3 studies) or one event called "influenza pandemic" (15 studies). Barnett et al. [28] examined four events: weather related, pandemic influenza, dirty bomb radiological terrorism, and anthrax bioterrorism. Cone et al. [29] also examined multiple disasters scenarios: earthquakes, ice storms, tornados, flood, fire rescue, hurricane, and biological, chemical, and radiation events. Hope et al. [30] similarly examined three scenarios: weather related pandemic, and bioterrorism. The presentation of multiple disparate events made it necessary to limit the description of each event scenario. Fifteen studies - Basta et al. [31], Balicer et al. [32], Balicer et al. [33], Barnettt et al. [34], Cowden et al. [35], Damery et al. [36], Daugherty et al. [37], Garnett et al. [38], Gershon et al. [16], Hope et al. [39], Imai et al. [40], Seale et al. [41], Stuart and Gillespie [42], Syrett et al. [43], and Tippett et al. [44] - examined willingness to report to work exclusively in an influenza pandemic but scenarios varied dramatically from study to study. For example, Basta et al. [31] examined both early and peak pandemic duties to assess willingness to report to work contingent on duties requiring direct face to face contact with infected patients. Peak pandemic with high risk duties of direct face-to-face contacted produced a substantially lower willingness to report to work of 56.2\%. Hope et al. [30] studied clinical nurse consultants, educations, and managers before and after a four-hour education intervention and four-day influenza pandemic exercise. Syrett et al. [43] examined willingness of personnel to respond to a mass causality event with a developing scenario. The scenario was a biological event that was nontransmissible with proven prophylaxis and treatment; then a biological event that was transmissible with only experimental prophylaxis and treatment. Before the severity of the incident was known, providers were twice as willing to report to work. Once identified as transmissible, less than $40 \%$ were willing to report to work. For the purposes of this research, percent willing to report to work was reported for the worst-case scenario when multiple pandemic scenarios were presented. Of these 18 studies, willingness to report to work varied from $27.00 \%$ (Gershon et al. [16]) to 93.10\% (Barnett et al. [34]), M=65.87. In Gershon et al. [16]) only $11 \%$ of healthcare aides and $37 \%$ of Registered Nurses were willing to 
take care of influenza patients. Three studies (Balicer et al. [33], Barnett et al. [28] and Barnett et al. [34]), including the study with the highest percent willing workers, utilized the Witte's Extended Parallel Process Model, using a threatand-efficacy based framework. All three studies examined willingness to report to work if required and also if asked but not required. These studies produced similar results with a relatively small range $(82.50 \%$ to $93.10 \%)$ in providers' willingness.

\subsection{Healthcare providers: gender and groups}

Participants' gender was reported in 24 studies. The majority of the studied providers were female, $71.21 \%$. Their willinness ranged from $34.10 \%$ [34] in study of paramedics and emergency medical technicians to $96.20 \%$ [21] in a study of community health nurses. Unfortunately only eight studies presented gender specific data on willingness to report to work.

A total of 204 different provider groups were cited in studies. After duplicates were removed, there were 148 provider groups. Descriptions of professions also varied widely. Nurses' perceptions were studied in 25 studies. Their authors described nurse participants as follows: nurses (9 studies), clinical nurse consultants, clinical nurse specialists, community nurses, early childhood nurses, ER nurses, licensed practical nurses, any nurse, nurse educators and nurse managers, nurse practitioners, nurse unit managers, nursing, nursing administration (2 studies), nursing staff ( 2 studies), nursing assistants (2 studies), nursing students with a lesser degree nursing credential enrolled in a bachelor degree program. Likewise, 17 different labels applied to physician or doctor groups: attending physicians, doctors (2 studies), dentists (2 studies), ER physicians, general practitioners, hospital doctors, house staff/residents or fellows, interns, consultants, internal Medicine House Staff, MD, DO/PhD, medical registrars, medical staff, medical staff specialists, practitioners, registrars ( 2 studies), and residents. The category of "other" was noted in 5 studies.

The greatest diversity in provider groups was found in studies of hospitals and healthcare systems. For example, Balicer et al. [32] initially listed 18 categories of providers spanning foodservice/linen to hospital staff, clinical staff, physicians and nurses. Categories were then collapsed to clinical and nonclinical staff. Irvin et al. [22] collapsed seven provider groups into three categories: doctors, nurses, and other. Several studies referred to general department-based groups, such as administration, maintenance, engineering, community health workers, laboratory services, occupational therapy, pharmacy and telecom to name a few. Finally, some authors used broad classifications of professionals, such as allied health professionals, clinical staff, non-clinical staff, clerical staff, management and supervisors.

In sum, researchers used a broad range of labels and inconsistent groupings to describe healthcare workers. The most widely studied provider groups were nurses and physicians, therefore, we examined their willingness in greater detail. 


\subsection{Percentage of nurses and physicians willing to report to work}

We took a closer look at nurses' and physicians' willingness to report to work during an influenza pandemic but the number of studies was small (13 and 7, respectively). Mean willingness to report to work for physicians was $73.64 \%$ (range $66 \%$ to $87 \%$ ) and for nurses was $59.82 \%$ (range $23.10 \%$ to $90.10 \%$ ).

Four studies included no other provider group but nurses. Their findings varied widely. In Wong et al. [21] $23.10 \%$ of community health nurses were willing to work during the pandemic while in Martin [18] 90.10\% of nurses were willing to do so. Both studies were completed during H1N1 pandemic influenza. In the remaining nine studies the mean percent of nurses willing to report to work was $63.67 \%$ and similar to the mean percent of all providers included in these nine studies $(62.28 \%)$.

\subsection{Methodological differences in measuring willingness}

Differences that could possibly affect the study findings included item wording, scale type, scale point labels, use of a midpoint or additional response. In half of the studies, researchers used a variety of Likert-type scales, such as from 1 to 4 and from 1 to 100 . Fourteen studies used binary checkboxes with yes vs. no, likely vs. unlikely, willing vs. not willing, or would work/respond/volunteer vs. would not work/respond/volunteer. Variations were noted in the presence of a scale midpoint, which may indicate neutrality, or in offering response options such as "do not know," "unsure/undecided" or "not applicable". Dichotomization methods for splitting those willing and not willing to report to work also varied. Some researchers included neutral or midpoint responses into the unwilling group while others excluded such responses from all subsequent calculations. Therefore, due to the different scaling of responses and dichotomization methods, comparisons and generalizations across studies should be made with great caution. It is possible that these methodological differences explain a great amount of variance in findings across the reviewed studies.

\section{Summary}

\subsection{Summary of evidence}

A systematic review of 28 studies on willingness of healthcare providers to report to work during a pandemic influenza was completed. Healthcare provider group definitions varied widely and gender-specific data was limited. Researchers primarily studied nurses and physicians but other provider groups were also included, as well as a grouping of all providers within a specific department.

In most studies, the authors failed to specify willingness to report to work by provider category. Therefore, it is too early to generalize provider-specific findings across available studies. The only exception is the comparison of physicians and nurses, which overall showed higher willingness to report to work among physicians, but even this finding should be interpreted with great 
caution because the number of studies was small and their methodologies varied widely.

\section{Practical implications for planning}

\subsection{Implications for planning and policy}

An influenza pandemic will place an enormous burden on the healthcare system; therefore, pre-pandemic planning is essential to reduce morbidity and mortality. An effective response begins with a healthcare workforce that can respond to the surge of patient and maintain the continuum of care. It is essential to have accurate data demonstrating provider willingness to report to work. Future researchers should make every effort to collect data on first responders, emergency and critical care workers, such as emergency and critical physicians, critical nurses and paramedics, whose availability has a strong effect on disaster response. Planning efforts must also take into consideration the number of providers who may be willing but unable to report to work due to illness; a potential absentee rate that may reach $40-50 \%$.

Overall, the 28 studies demonstrate great variability in results from a low of $23.10 \%$ study (of community health nurses) during the $2009 \mathrm{H} 1 \mathrm{~N} 1$ pandemic) to a high of $93.10 \%$ in the study of EMS providers. Given the current state of the empirical literature, worst case scenario planners may be advised to adopt an estimate of $23 \%$ of healthcare providers willing to report to work, which could be further adjusted to account for those who are unable to do so due to being ill. If adjusted to reflect the number of providers inflicted with the influenza virus, the estimated percent of providers who are willing and able to perform their duties may fall below $15 \%$, which would have tremendous implications for workforce planning. Because the worst-case evidence comes from the study of nurses, the estimate of $15 \%$ may be adjusted upward for physicians who tend to express higher willingness to report to work than nurses.

Though training exercises are mandated by regulatory agencies and are aimed at enhancing response and mitigating the loss of life, limited data is available on the effectiveness of this training and its role in changing provider willingness to report to work. Therefore, a conservative planning approach will incorporate the worst-case scenario while taking into account the possibility of absenteeism due to illness. Meanwhile training programs and other interventions that enhance willingness to report to work must be implemented. Successful programs must be shared with disaster response, academic, and regulatory communities in order to prioritize funding, education, training, and ultimately improve disaster response.

\subsection{Limitations}

The absence of healthcare sector information, gender, and worker group limited the ability to draw generalizations on willingness to report to work. The studies were limited to those published in English. Only studies published as of May 
2011 were included in the review. This analysis was limited to studies conducted for the purpose of evaluating willingness to report to work in a pandemic influenza. This review did not explore internal or external incentives, or interventions associated with willingness to report to work.

\subsection{Conclusions}

Our review showed large variations in healthcare providers' willingness to report to work during a flu pandemic. While it is too early to generalize with confidence, a worst-case scenario planning approach was suggested based on the empirical evidence available to us today. Future researchers are encouraged to include tables that show willingness to report to work by healthcare sector, worker type, and gender. For disaster planning analysis and comparison it is essential that worker types be explained. Cowden et al. [35] serves as a model providing a detailed description of occupational categories that would be relevant to many researchers. Future researchers should carefully plan their scenario design and make every effort to study emergency and critical care physicians, critical care nurses and paramedics who are at the frontline of providing care during a pandemic outbreak. Barnett et al. [34] and Tippett et al. [44] provide excellent examples of EMS providers' willingness to report to work. Finally, research-practitioner collaborations during a real-life influenza pandemic event are perhaps the best opportunities to estimate willingness while minimizing social desirability bias.

\section{References}

[1] CDC Estimates of 2009 H1N1 Influenza Cases Hospitalizations and Deaths in the United States, April 2009 - April 10, 2010; Center for Disease Control (CDC). http://www.cdc.gov/h1n1flu/estimates_2009 hln1.htm

[2] Chan M. H1N1 in Post-pandemic Period. World Health Organization 2010 http://www.who.int/mediacentre/news/statements/2010/h1n1_vpc_201008 10/en/index.html\#

[3] The Role of WHO and Recommendations for National Measures Before and During Pandemics. WHO Global Influenza Preparedness Plan; World Health Organization (WHO). www.who.int/csr/resources/publications/ influenza/en/WHOCDSCSRGIP20055.pdf

[4] The Influenza Pandemic of 1918. Virus: Stanford University. www.virus.stanford.edu/uda/

[5] Public Health Emergency Response: A Guide for Leaders and Respondents; U.S. Department of Health and Human Services (USDHHS) www.phe.gov/emergency/communication/guides/leaders/Documents/freo5 08final.pdf

[6] Barry, J. M. The Great Influenza. Penguin Books: London, pp. 4-5, 2005. 
[7] HHS Pandemic Influenza Plan; U.S. Department of Health and Human Services (USDHHS) www.hhs.gov/pandemicflu/plan/pdf/HHSPandemic InfluenzaPlan.pdf

[8] Pandemic Influenza Preparedness Response and Recovery Guide for Critical Infrastructure and Key Resources; U.S. Department of Homeland Security (USDHS). www.nh.gov/readynh/business/documents/critical infrastructurepandemicinfluenzaguide1.pdf

[9] Altered Standards of Care in Mass Casualty Events. Bioterrorism and Other Public Health Emergencies; Agency for Healthcare Research and Quality (AHRQ). www.archive.ahrq.gov/research/altstand

[10] Pandemic Influenza Preparedness and Response Guidance for Healthcare Workers and Healthcare Employers; Occupational Safety and Health Administration (OSHA). www.osha.gov/Publications/3328-05-2007English.html

[11] Report on the National Disaster Health Workforce. National Center for Disaster Medicine and Public Health (NCDMPH). Rockville, MD.www.usuhs.mil/pdf/NCDMPHWorkforceProject2011-B.pdf

[12] National Strategy for Pandemic Influenza Implementation Plan. Homeland Security Council. The White House. www.georgewbushwhitehouse.archives.gov/homeland/pandemic-influenzaimplementation.html

[13] Kilpatrick, A. M., Chmura, A. A., Gibbons, D. W., Fleischer, R. C., Marra P. P., Daszak, P., Predicting the global spread of H5N1 avian influenza. PNAS. 103(51), pp.19368-19373, 2006.

[14] Cumulative Number of Confirmed Human Cases for Avian Influenza A(H5N1) Reported to WHO, 2003-2011; World Health Organization. www.who.int/influenza/human_animal_interface/EN_GIP_20120105Cum ulativeNumberH5N1 cases.pdf

[15] Chaffee, M., Willingness of healthcare personnel to work in a disaster: An integrative review of the literature. Disaster Medicine and Public Health Preparedness, 3(1), pp. 42-56, 2009.

[16] Gershon, R. R., Magda, L. A., Canton, A. N., Riley, H. E. M., Wiggins, F., Young, W., and Sherman, M. F. 2010. Pandemic-related ability and willingness in home healthcare workers. American Journal of Disaster Medicine, 5(1), pp. 15-26, 2010.

[17] Shi, L., Health Services Research Methods. (2nd ed.). Thompson: Clifton Park, New York, 2008.

[18] Martin, S.D., Nurses ability and willingness to work during pandemic flu. Journal of Nursing Management, 19, pp. 98-108, 2011.

[19] Saleh, D. A., and Elshaer, I., Nurses' perspectives and concerns towards an infectious disease epidemic in Egypt. The Egyptian Journal of Community Medicine, 28(2), pp. 1-17, 2010.

[20] Ma, X., He, Z., Wang, Y., Jiang, L., Xu, Y., Qian, C., Sun, R., Chen, E., Hu, Z., Zhou, L., Zhou, F., Qin, T., Cao, X., An, Y., Sun, R., Zhang, X., Lin, J., Ai, Y., Wu, D., Du, B., and The China Critical Care Clinical Trial Group, Knowledge and attitudes of healthcare workers in Chinese 
intensive care units regarding $2009 \mathrm{H} 1 \mathrm{~N} 1$ influenza pandemic. $B M J$ Infectious Diseases, 11(24), 2011.

[21] Wong, E. L. Y., Wong, S. Y. S, Kung, K., Cheung, A. W. L, Gao, T. T., and Griffiths, S., Will the community nurses continue to function during H1N1 influenza pandemic: a cross-sectional study of Hong Kong community nurses? BioMed Central Health Services Research, 10(107), 2010.

[22] Irvin, C. B., Cindrich, L., Patterson, W., and Southall, A., Survey of hospital healthcare personnel response during a potential avian influenza pandemic: will they come to work? Prehospital Disaster Medicine, 23(4), pp. 328-335, 2008.

[23] Martinese, F., Keijzers, G., Grant, S., and Lind, J., How would Australian hospital staff react to an avian influenza admission, or an influenza pandemic? Emergency Medicine Australasia, 21(1), pp.12-24, 2009.

[24] Shabanowitz, R. B., and Reardon, J. E., Avian flu pandemic-flight of the healthcare worker? HEC Forum, 21(4), pp. 365-385, 2009.

[25] Tzeng, H. M., and Yin, C. Y., Nurses' fears and professional obligations concerning possible human-to-human avian flu. Nursing Ethics, 13(5), pp.455-470, 2006.

[26] Schechter, S., Medical reserve corps volunteers' ability and willingness to report to work for the Department of Health during catastrophic disasters. Unpublished master thesis, Monterey, CA: Naval Postgraduate School, 2007.

[27] Wong, T. Y., Koh, G. C., Cheong, S. K., Sundram, M., Koh, K., Chia, S. E., and Koh, D., A cross-sectional study of primary-care physicians in Singapore on their concerns and preparedness for an avian influenza outbreak. Annals of the Academy of Medicine, Singapore, 37(6), pp. 458464, 2008.

[28] Barnett, D. J., Balicer, R. D., Thompson, C. B., Storey, J. D., Omer, S. B., Semon, N. L., Bayer, S., Cheek, L. V., Gateley, K. W., Lanza, K. M., Norbin, J. A., Slemp, C. C., and Links, J. M., Assessment of local public health workers' willingness to respond to pandemic influenza through the application of the extended parallel process model. PLoS One, 4(7), 2009.

[29] Cone, D. C., and Cummings, B. A., Hospital disaster staffing: If you call, will they come? American Journal of Disaster Medicine, 1(1), pp. 28-36, 2006.

[30] Hope K., Durrheim, D., Barnett, D., D’Este, C., Kewley, C., Dalton, C., White, N., Kohlhagen, J., and Links, J., Willingness of frontline health care workers to work during a public health emergency. Australian Journal of Emergency Management, 25(3), pp. 39-47, 2010.

[31] Basta, N. E., Edwards, S. E., and Schulte, J., Assessing public health department employees' willingness to report to work during an influenza pandemic. Journal of Public Health Management Practices, 15(5), pp. 375-383, 2009. 
[32] Balicer, R. D., Omer, S. B., Barnett, D. J., and Everly, G. S., Local public health workers; perceptions towards responding to an influenza pandemic. BioMed Central Public Health, 6, 2006. http://archive.ahrq.gov/research/altstand.

[33] Balicer, R. D., Barnett, D. J., Thompson, C. B., Hsu, E. B., Catlett, C. L., Watson, C. M., Semon, N. L., Gwon, H. S., and Links, J. M., Characterizing hospital workers' willingness to report to duty in an influenza pandemic through threat-and efficacy-based assessment. BioMed Central Public Health, 10, 2010.

[34] Barnett, D. J., Levine, R., Thompson, C. B., Wijetunge, G. U., Oliver, A. L., Bentley, M. A., Neubert, P. D., Pirrallo, R. G., Links, J. M., and Balicer, R. D., Gauging U.S., emergency medical services workers' willingness to respond to pandemic influenza using a threat- and efficacybased assessment framework. PLoS One, 5(3), 2010.

[35] Cowden, J., Crane, L., Lezotte, D., Glover, J., and Nyquist, A., Pre-pandemic planning survey of healthcare workers at a tertiary care children's hospital: ethical and workforce issues. Influenza Other Respiratory Viruses, 4(4), pp. 213-222, 2010.

[36] Damery, S., Wilson, S., Draper, H., Gratus, C., Greenfield, S., Ives, J., Parry, J., Petts, J., and Sorell, T., Will the NHS continue to function in an influenza pandemic? A survey of healthcare workers in West Midlands, UK. BioMed Central Public Health, 9, 2009.

[37] Daugherty E. L., Perl, T. M., Rubinson, L., Bilderback, A., and Rand, C. S., Survey study of the knowledge, attitudes, and expected behaviors of critical care clinicians regarding and influenza pandemic. Infection Control and Hospital Epidemiology, 30(12), pp.1143-1149, 2009.

[38] Garnett, A. L., Park, S. Y., and Redlener, I., Mitigating absenteeism in hospital workers during a pandemic. Disaster Medicine and Public Health Preparedness, 3(2), pp. S141-S147, 2009.

[39] Hope K., Massey, P. D., Osbourn, M., Durrheim, D. N., Kewley, C. D., and Turner, C., Senior clinical nurses effectively contribute to the pandemic influenza public health response. Australian Journal of Advanced Nursing, 28(3), pp. 47-52, 2009.

[40] Imai, T., Takahashi, K., Todoroki, M., Kunishima, H., Hoshuyama, T., Ide, R., Kawasaki, T., Koyama, N., Endo, K., Fujita, H., Iwata, K., Koh, G., Chia, S. E., and Koh, D., Perception in relation to a potential influenza pandemic among healthcare workers in Japan: Implications for preparedness. Journal of Occupational Health, 50, pp.13-23, 2008.

[41] Seale, H., Leask, J., Po, K., and MacIntyre, C.R., Will they just pack up and leave? - Attitudes and intended behaviors of hospital health care workers during and influenza pandemic. BioMed Central Health Services Research, 9(30), pp.1-8, 2009.

[42] Stuart, R. L., and Gillespie, E., Preparing for an influenza pandemic: healthcare workers' opinions on working during a pandemic. Healthcare Infection, 13, pp. 95-99, 2008. 
[43] Syrett, J. L., Benitez, J. G., Livingston, W.H., and Davis, E. A., Will emergency health care providers respond to mass causality incidents? Prehospital Emergency Care, 11, pp.49-54, 2007.

[44] Tippett, V. C., Watt, K., Raven, S. G., Psych (Hons) B., Kelly, H. A., Coory, M., Archer, F., and Jamrozik, K., Anticipated behaviors of emergency prehospital medical care providers during an influenza pandemic. Prehospital Disaster Medicine, 25(1), pp.20-25, 2010. 